\title{
Rapid Detection of Vancomycin-Resistant Enterococci (VRE) in Rectal Samples from Patients Admitted to Intensive Care Units
}

\begin{abstract}
Pedro Alves d’Azevedo ${ }^{1,3}$, Kelly Aline de Souza Santiago ${ }^{1}$, Guilherme Henrique Campos Furtado ${ }^{2}$, Diego Batista Xavier ${ }^{4}$, Antonio Carlos Campos Pignatari ${ }^{1}$ and Ricardo Titze-de-Almeida ${ }^{4}$

${ }^{1}$ Laboratório Especial de Microbiologia Clínica - LEMC/ALERTA, Universidade Federal de São Paulo - UNIFESP; ${ }^{2}$ Comissão de Epidemiologia Hospitalar, Universidade Federal de São Paulo - UNIFESP; ${ }^{3}$ Laboratório de Cocos Gram-positivos, Universidade Federal de Ciências da Saúde

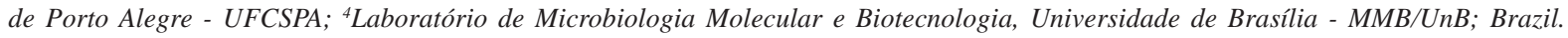

\begin{abstract}
The reduction in time required to identify vancomycin-resistant enterococci (VRE) has gained increased importance during hospital outbreaks. In the present study, we implemented a laboratory protocol to speed up the VRE screening from rectal samples. The protocol combines a medium for selective VRE isolation (VREBAC ${ }^{\circledR}$, Probac, São Paulo) and a multiplex PCR for detection and identification of vanA and vanB resistance genes. The screening performance was analyzed in $\mathbf{1 1 4}$ specimens collected from four intensive care units. The swabs were collected at two periods: (1) during a VRE outbreak (February 2006, $n=83$ patients) and (2) at the post-outbreak period, after adoption of infection control measures (June 2006, $n=31$ patients). Forty-one/83 VRE (49.4\%) and 3/31(9.7\%) VRE were found at the first and second period, respectively. All isolates harbored the van $A$ gene. In both periods, detection of the gene vanA parallels to the minimum inhibitory concentration values of $>256 \mu \mathrm{g} / \mathrm{mL}$ and $>48 \mu \mathrm{g} / \mathrm{mL}$ for vancomycin and teicoplanin, respectively. Multiplex PCR and conventional methods agreed in $\mathbf{9 0 . 2 \%}$ for enterococci identification. Besides this accuracy, we also found a remarkable reduction in time to obtain results. Detection of enterococcal species and identification of vancomycin resistance genes were ready in 29.5 hours, in comparison to 72 hours needed by the conventional methods. In conclusion, our protocol identified properly and rapidly enterococci species and vancomycin-resistance genes. The results strongly encourage its adoption by microbiology laboratories for VRE screenning in rectal samples.

Key-Words: Enterococcus, VRE, vancomycin, diagnostic, PCR.
\end{abstract}

Vancomycin-resistant enterococci (VRE) have shown a remarkable ability to spread during hospital outbreaks [1,2]. Specific control measures need the proper identification of individuals colonized by VRE through surveillance cultures $[3,4]$. The screening for VRE in rectal samples involves the use of selective isolation media, like the bile esculin azide agar with vancomycin $6 \mu \mathrm{g} / \mathrm{mL}$ [5]. Complementary tests to identify the genus Enterococcus and to confirm the resistance phenotype are required for conclusive results. Also, the intrinsic vanC-type species E. casseliflavus and E. gallinarum should be distinguished from the other species that carry the highlevel vancomycin-resistance genes vanA or vanB [6]. Biochemical tests for identification are time-consuming, expensive and they are sometimes inconclusive. Conventional protocols to detect vancomycin resistance as the disk diffusion test, Etest, and broth microdilution are time-consuming as well [7]. The automated methods also have some drawbacks. They need expensive equipments and materials and are highly accurate only for $E$. faecalis and E. faecium identification $[8,9]$.

Until recently, molecular biology techniques such as the polymerase chain reaction (PCR) were restricting to university laboratories. However, those equipments had become cheaper

Received on 12 May 2009; revised 19 July 2009.

Address for correspondence: Dr. Pedro A. d' Azevedo. Rua Sarmento Leite 245/204. Porto Alegre, RS, Brazil. Zip Code 90050-170. Phone: +55-51-33038740. Fax: +55-51-33039000. Email: pedro_dazevedo@yahoo.com.br. Financial support: CNPq, LEMC, UFCSPA.

The Brazilian Journal of Infectious Diseases

2009;13(4):289-293. (C) 2009 by The Brazilian Journal of Infectious Diseases and Contexto Publishing. All rights reserved. and various reagent kits are commercially available. Nowadays clinical laboratories have introduced the PCR technique for routine diagnostic testing [10]. Thus, rapid and accurate methods to identify microbial species, with low cost and applicable to routine laboratory practices have been developed. The present study implemented a rapid method to detect VRE in rectal swabs from ICU patients by using the selective VREBAC ${ }^{\circledR}$ medium and a multiplex PCR scheme that identify enterococci species and vancomycin-resistance genes.

\section{Materials and Methods}

Hospital Setting and Study Design

We examined rectal samples from intensive care units (ICUs) from São Paulo Hospital, a 700-bed university affiliated hospital in São Paulo, Brazil. The specimens were collected from all eligible patients at four ICUs (Medical, Medicalsurgical, Pneumonology, and Adult Emergency). Our study adopted the following inclusion criteria for swab collection: long-stay in hospital; previous antimicrobial therapy (mainly vancomycin); severity of illness; imunossupression; previous abdominal or cardiothoracic surgery, and invasive procedures. Eighty-three specimens were examined for the presence of VRE at the first period (during the outbreak, February, 2006). Thirty-one specimens were examined at the second period (the post-outbreak period, June, 2006). At the later period, we measured the time spent for each activity from the sample collection until species and resistance determination.

Specimen Collection and Broth Cultures

The specimens were collected by nurses according to Infection Control Committee protocols. Briefly each swab was 
carefully introduced in the anal sphincter region $(1-5 \mathrm{~cm})$ and gently rotated. Immediately after, it was immersed in saline solution $(0.9 \%, 5 \mathrm{~mL})$ and transported to the microbiology laboratory. In the laboratory this diluted faecal samples were cultured in all three VREBAC ${ }^{\circledR}$ media. Medium I contained agar azide and the selective antimicrobials aztreonam, polymyxin B and amphotericin B. The medium II contained vancomycin $6 \mu \mathrm{g} / \mathrm{mL}$. Medium III had the same medium II antimicrobial and a chromogenic marker (Chromagar Orientation $^{\circledR}$ ) [11]. VREBAC ${ }^{\circledR}$ laminocultures were incubated at $35^{\circ} \mathrm{C}$ for $18-24$ hours.

Species Identification by Conventional Methods

To identify the genus Enterococcus, we used the following phenotypic tests: catalase, $\mathrm{NaCl} 6.5 \%$ broth, agar bile esculin azide, and L-pyrrolidonyl- $\beta$-naphthylamide hydrolysis (PYR test). The biochemical tests used to identify enterococci species was Purple Broth Base (Difco, Detroit, USA) supplemented with arabinose, MGP (Metil- $\alpha$-Dglycopiranoside), arginine, mannitol, sorbitol, sorbose and raffinose. Motility and pigmentation were also determined [12].

\section{Multiplex Polymerase Chain Reaction Scheme}

We used a multiplex PCR scheme that identifies the species E. faecalis, E. faecium, E. gallinarum (vanC1), and E. casseliflavus (vanC2/3). This method also detects, in the same reaction, the resistance genes van $A$ and vanB (Table 1 ). The primer concentrations previously adjusted to provide easily distinguished bands were $d d l_{E \text {.faecalis }}, d d l_{E \text {.faecium }}$, E. gallinarumvanC1, E. casseliflavus-vanC2/3 (18 pmol of each primer), vanA (3 pmol of each primer), and vanB (2 pmol of each primer) [13]. For each PCR reaction, 5 - 10 colonies were picked up from the chromogenic medium II. The PCR mixture contained the primers and the following reagents: Taq Buffer $1 \mathrm{X}, 3 \mathrm{mM}$ of $\mathrm{MgCl}_{2}, 0.25 \mathrm{mM}$ of each dNTP, and 2U of Taq DNA polimerase. The amplification cycles were: initial preheating step at $94^{\circ}$ for $2 \mathrm{~min}$; initial denaturation step at $94^{\circ} \mathrm{C}$ for $5 \mathrm{~min}$; 30 cycles of amplification (denaturation $94^{\circ} \mathrm{C}$ for $1 \mathrm{~min}$, annealing at $55^{\circ} \mathrm{C}$ for $1 \mathrm{~min}$, and extension at $72^{\circ} \mathrm{C}$ for $2 \mathrm{~min}$ ); and a final extension at $72^{\circ} \mathrm{C}$ for $5 \mathrm{~min}$.

The PCR products were electrophoresed through a $1 \%$ agarose gel stained with ethidium bromide for $40 \mathrm{~min}$ at $60 \mathrm{~V}$ and photographed under UV light.

\section{Vancomycin and Teicoplanin Susceptibility}

We determined the antimicrobial susceptibility to

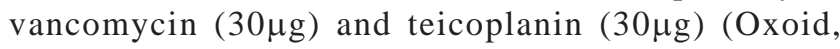
Basingstoke, UK) using the disk diffusion method (KirbyBauer), according to the NCCLS guidelines [14]. The plates were incubated at $35^{\circ} \mathrm{C}$ for 18 - $24 \mathrm{~h}$. The resistant isolates were submitted to E-test ${ }^{\circledR}$ according to the manufacturer recommendations to determine their minimal inhibitory concentration (MIC) to vancomycin and teicoplanin. The quality control strains used in our study were ATCC E. faecalis 29.212 and E.coli 24.922.

\section{Results}

Species Identification and Vancomycin Resistance Results

We first analyzed the colony growing in each VREBAC ${ }^{\circledR}$ laminoculture medium I, II, and III. At the first time point (during the outbreak), all enterococci isolates ( $n=83,100 \%)$ grew in the PROBAC ${ }^{\circledR}$ medium I. From them, 18 (21.7\%) isolates grew only in this medium and they showed susceptibility to vancomycin. The medium II, which contained vancomycin $6 \mu \mathrm{g} / \mathrm{mL}$, showed a lower number of positive growing $(n=65$, 78.3\%). Six vancomycin susceptible enterococci (VSE) surpassed the antimicrobial inhibitory effects and grew in this media. The chromogenic and selective medium III identified 59 positive isolates (71.1\%). Enterococci isolates were easily distinguished from the other microbial species owing to the light green coloration of their colonies

All isolates with positive growing in all three VREBAC ${ }^{\circledR}$ media ( $n=59$ ) were selected for the following confirmatory tests: identification of the genus Enterococcus, resistance to vancomycin and multiplex PCR. Nine of the 59 isolates (15.2\%) showed susceptibility to vancomycin in the disk diffusion test, thus they were false positive VRE; all of them showed a lower number of colonies (2 - 6) in the medium III.

The multiplex PCR identified the species E. faecium (n=20; $48.8 \%$ ) and $E$. faecalis ( $\mathrm{n}=21 ; 51.2 \%$ ). They were all positive for the gene vanA. The rate of agreement between PCR and conventional biochemical tests was $90.2 \%$. The divergent results occurred for 4 isolates (9.8\%) which had the $E$. faecalis $d d l$ gene amplified by PCR but showed E. faecium biochemical profiles.

At the second period, we analyzed 31 specimens. From them, only 4 isolates grew in the chromogenic medium III. Conventional biochemical tests and PCR identified the species E. faecium. Three of them carried the vanA gene. The false positive VRE also showed a reduced number of colonies $(n=3)$ in the medium III.

Our results showed that the selective and chromogenic medium III provided the best accurate outcome for the presumptive diagnostic of VRE. With regard to vancomycin resistance, the identification of the vanA gene by the multiplex PCR was consistent with to the disk diffusion testing findings. In addition, this VanA genotype agreed with the MIC values determined for vancomycin $(>256 \mu \mathrm{g} / \mathrm{mL})$ and teicoplanin $(>48$ $\mu \mathrm{g} / \mathrm{mL})$ by Etest method.

\section{Time to Obtain Results}

The proposed protocol for VRE screening from rectal samples was able to reduce the time spent to identify species and vancomycin resistance. The protocol consumed 29.5 hours for all the laboratorial steps from the specimen collection to the final result (Table 2). We identified two time-consuming activities among the procedures grouped in the step 1, the 5.5 hours between the rectal swab collection and the VREBAC ${ }^{\circledR}$ culture. They were the time to deliver the swabs to the laboratory and further registration (3h) and the period of incubation in saline solution (2h). The second step for VRE screening comprised the VREBAC ${ }^{\circledR}$ culture procedures. The 
Table 1. Oligodeoxynucleotide primers

\begin{tabular}{|c|c|c|c|}
\hline Amplified gene & Sequence $\left(5^{\prime}-3^{\prime}\right)^{a}$ & Amplicon size (bp) $)^{b}$ & Reference \\
\hline \multirow{2}{*}{$d d l_{E \text {. faecalis }}$} & F:ATCAAGTACAGTTAGTCT & & \\
\hline & R:ACGATTCAAAGCTAACTG & 941 & 21 \\
\hline \multirow{2}{*}{$d d l_{\text {E. faecium }}$} & F:TAGAGACATTGAATATGCC & & \\
\hline & R:TCGAATGTGCTACAATC & 550 & 21 \\
\hline \multirow[t]{2}{*}{ E. gallinarum (vanC1) } & F: GGTATCAAGGAAACCTC & & \\
\hline & R: CTTCCGCCATCATAGCT & 822 & 21 \\
\hline \multirow[t]{2}{*}{ E. casseliflavus (vanC2/3) } & F:CTCCTACGATTCTCTTG & & \\
\hline & R:CGAGCAAGACCTTTAAG & 439 & 21 \\
\hline \multirow[t]{2}{*}{ vanA } & F: GGGAAAACGACAATTGC & & \\
\hline & R:GTACAATGCGGCCGTTA & 732 & 21 \\
\hline \multirow[t]{2}{*}{$\operatorname{van} B$} & F:ACCTACCCTGTCTTTGTGAA & & \\
\hline & R:AATGTCTGCTGGAACGATA & 300 & 24 \\
\hline
\end{tabular}

${ }^{a} \mathrm{~F}$, sense primer; R, antisense primer. ${ }^{\mathrm{b}}$ Size of the amplified gene for enterococci identification and vancomycin resistance.

Table 2. Time for each activity from the VRE screening protocol

\begin{tabular}{lc}
\hline Activities & Time \\
\hline Step 1. Specimens collection and incubation in saline & \\
1.1 Collection of swabs in the ICUs & $40^{\prime}$ \\
1.2 Time to deliver the samples to the microbiology laboratory & $2.3 \mathrm{~h}$ \\
1.3 Registration of samples & $30^{\prime}$ \\
1.4 Swab incubation in saline solution & $2 \mathrm{~h}$ \\
Subtotal & $5.5 \mathrm{~h}$ \\
Step 2. VREBAC ${ }^{\circledast}$ culture & \\
2.1 Culture in VREBAC ${ }^{\circledR}$ media & $1 \mathrm{~h}$ \\
2.2 Incubation of the VREBAC ${ }^{\circledR}$ media & $18 \mathrm{~h}$ \\
2.3 Selection of presumptive VRE isolates & $10^{\prime}$ \\
Subtotal & $19.2 \mathrm{~h}$ \\
Step 3. PCR reaction and electrophoresis & \\
3.1 Transport of VREBAC ${ }^{\circledR}$ to the molecular biology laboratory & $10^{\prime}$ \\
3.2 Preparation of the PCR reactions & $40^{\prime}$ \\
3.3 PCR amplification cycles & $3 \mathrm{~h}$ \\
3.4 Agarose gel preparation & $15{ }^{\prime}$ \\
3.5 Electrophoresis & $40^{\prime}$ \\
Subtotal & $4.8 \mathrm{~h}$ \\
Total time & $29.5 \mathrm{~h}$ \\
\hline
\end{tabular}

selective chromogenic medium III contributed to shorten the time for obtainining the results, enabling to visualize presumptive VRE colonies at 18 hours. Additionally the multiplex PCR also contributed to reduce the time to obtain results. The method was able to identify VRE species and vancomycin resistance genes in 4.8 work hours.

\section{Discussion}

Vancomycin-resistant enterococci are infectious agents with remarkable ability to spread and cause hospital outbreaks. The most commonly reported risk factors for VRE infection are long stay in hospital, use of vancomycin and third-generation cephalosporins, and chronic dialysis $[15,16,17]$. Patients chronically admitted to ICU are at high risk for colonization and outbreaks commonly occur in such hospital wards [1].
In our study, we found a high rate of VRE carriage (49.4\%) during the outbreak occurred at the ICUs of São Paulo Hospital.

The VRE prevalence in 126 adult ICUs from 60 US hospitals enrolled in the National Nosocomial Infections Surveillance (NNIS) was extensively analyzed [18]. The prevalence rate varied among the hospitals $(0-59 \%)$ and was higher at major teaching centers or larger hospitals (with $>500$ beds), similar to our hospital.

Our study identified a great decrease in the number of positive VRE specimens from $49.4 \%$ to $9.7 \%$ after adoption of infection control measures. The screening protocol adequately identified VRE isolates during the outbreak, when the bacterial colonization is higher. Furthermore, the same protocol was sensitive to detect VRE at lower prevalence rates in the post- 
outbreak period. High sensitivity would be important to detect epidemic strains during non-outbreak periods or to conduct surveillance studies in non-ICU hospital wards. Interventions aimed to reduce the VRE prevalence in non-ICU areas are valuable to diminish the risk of VRE colonization in ICU [18]. The isolates showed different ability to growth in each VREBAC ${ }^{\circledR}$ laminoculture media I, II, and III. The medium I contains selective antimicrobials (aztreonam, polymyxin B, and anfotericin B) that suppress the overgrowth of various species from the intestinal microbiota. However, our group and others have observed that some nonenterococcal species (Pseudomonas, Klebsiella, Candida, and Streptococcus) surpass the inhibitory effects and grow in this selective media [5]. Thus, we pre-incubated the rectal swab in $5 \mathrm{~mL}$ of $0.9 \%$ saline solution for two hours before VREBAC ${ }^{\circledR}$ culture to reduce the growing of false positive isolates. With regard to medium II, six vancomycin susceptible enterococci surpassed the inhibitory effects of vancomycin $6 \mu \mathrm{g} / \mathrm{mL}$. Previous study, using media contained vancomycin $6 \mu \mathrm{g} / \mathrm{mL}$ also found the growing of vancomycin-susceptible E. faecium and E. faecalis [19].

The use of chromogenic medium has provided satisfactory results for VRE screening directly from faecal samples. In our study, the chromogenic medium III allowed to distinguish enterococci from other species owing to the typical light green coloration. In this chromogenic VRE selective medium, 59 isolates (71.1\%) showed positive growing. In a recent study, the authors tested another chromogenic media named chromID VRE (Biomérieux, Marcy-l'Etoile, France). This media was able to select for and distinguish between vancomycin-resistant E. faecalis and vancomycin-resistant E. faecium [20]. This valuable discriminatory property is still not provided by the VREBAC ${ }^{\circledR}$ medium. However, the species E. faecium and $E$. faecalis were rapidly identified (4.8 work hours) by our multiplex PCR scheme. Our method may distinguish, in addition, the VanA-, VanB-, and VanC-resistance genotypes, a feature still not provided by any chromogenic medium.

Various PCR protocols have been described to identify enterococci species and to detect vancomycin-resistance genotypes $[7,21,22,23]$. The method used in our study allows the simultaneous identification of E. faecium, $E$. faecalis, E. casseliflavus, and E. gallinarum sequences, as well as the vancomycin-resistant genes vanA and $\operatorname{van} B$ in one unique reaction [13]. The scheme used primers previously described and provided easily visualized bands after agarose gel electrophoresis [21,24]. It also reduced the time usually spent for DNA extraction because the colonies are picked up directly from the plate to the PCR mixture. The rate of agreement between this PCR scheme and the conventional biochemical assays was high for all species tested, eg., E. faecalis (95\%), E. faecium (95\%), E. gallinarum (100\%) and E. casseliflavus (100\%) [13]. In the present study, only 4 isolates (9.8\%) showed discordance between PCR and the biochemical testing. As far as we know, the present study was the first to test this PCR scheme during a VRE outbreak.
Regarding to resistance genes, all E. faecium and E. faecalis isolates carried the $v a n A$ gene. This high-level of vancomycin resistance was also found in both E. faecalis and E. faecium isolated from patients from other Brazilian hospital, during the first Brazilian VanA outbreak [24].

The rapid identification of VRE is highly valuable during hospital outbreaks $[4,16]$. Thus, we carefully evaluated the time spent in each VRE screening activity of our protocol. We identified a delay of 2.8 hours between the swab collection and the specimen incubation in saline solution. Such activity should consume no more than 30 minutes (Table 2, items 1.2 and 1.3). The incubation in saline should also be shortened to 1 hour without compromising its inhibitory action (item 1.4). Finally, whether the individuals responsible for PCR were previously notified when the VREBAC culture was ready, they could left the PCR mixture prepared, which would save forty minutes (Table 2, item 3.2). Also, the agarose gel preparation may occur during the $3 \mathrm{~h}$ amplification cycles. Thus, a better planning and reinforcement on the role of each participant would save at least 4.2 hours. The final screening report on the species and vancomycin resistance genes would be provided in 25.3 hours. Previous publications have also described rapid protocols for VRE screening. The use of real-time PCR directly from the rectal swab provided results in $4-5$ hours but the sensitivity was lower in comparison with the cultured samples [25]. In addition, the high cost of the real-time PCR equipment is incompatible with the general microbiology laboratories.

\section{Conclusion}

The present study evaluated a protocol for VRE screenning in rectal samples from patients admitted to ICU. It employed the selective medium VREBAC ${ }^{\circledR}$ and the multiplex PCR allowing reporting enterococci species and vancomycin resistance genes in up to 29.5 hours. The method showed sensitivity to detect VRE in high and low prevalence rates. It provided highly concordant results compared to conventional biochemical and susceptibility testing. The results found in our study encourage microbiology laboratories to adopt this rapid protocol for VRE screening during hospital outbreaks.

\section{References}

1. Patel, R. Clinical impact of vancomycin-resistant enterococci. J Antimicrob Chemother 223; 51(S3):12-21.

2. Top, J.; Willems, R.; Bonten, M. Emmergence of CC17 Enterococcus faecium: from commensal to hospital-adapted pathogen. FEMS Immunol Microbiol 2008; 52:297-308.

3. Centers for Disease Clinical and Prevention - CDC. Recommendations for preventing the spread of vancomycin resistance: recommendations of the Hospital Infection Clinical Practices Advisory Committee (HICPAC). United States, 1995. M.M.W.R. 1995; 44 (RR-12): 1-13.

4. Tacconelli, E.; Cataldo, M.A. Vancomycin-resistant enterococci (VRE): transmisssion and control. Int J Antimicrob Agents 2008; 31:99-106.

5. Van Horn, K.G.; Gedris, C.A.; Rodney, K. M. Selective isolation of vancomycin-resistant enterococci. J Clin Microbiol 1996; 34:924-7. 
6. Toye, B.; Shymanski, J.; Bobrowska, M. et al. Clinical and epidemiologic significance of enterococci intrinsically resistant to vancomycin (possessing the Vanc genotype). J Clin Microbiol 1997; 35:3166-70.

7. Domig, K.J.; Mayer, H.K.; Kneifel, W. Methods used for the isolation, enumeration, characterization and identification of Enterococcus spp. 2. Pheno- and genotypic criteria. Int J Food Microbiol 2003; 88:165-188.

8. d’Azevedo P.A., Dias C.A.G., Gonçalves A.L.S., Rowe F., Teixeira L.M. Evaluation of an automated system for the identification and antimicrobial susceptibility testing of enterococci. Diag Microbiol Infect Dis 2001; 40:157-161.

9. d'Azevedo P.A., Cantarelli V., Inamine E., Superti S., Dias C.A.G. Avaliação de um sistema automatizado na identificação de espécies de Enterococcus. J Bras Patol Med Lab 2004; 237-9.

10. Versalovic J.; Lupski J. Molecular detection and genotyping of pathogens: more accurate and rapid answers. Trends Microbiol 2002; 10:S15-21.

11. Martino M.D.V., Ravo-Osorio L.M., Mímica L., Garcia C., Silva C. B., Sasagawa S.M., Mímica I.M. Screening for glycopeptide vancomycin resistant fecal carriers. ASM General Meeting Chicago, may 1999;

12. Facklam R.R., Teixeira L.M. Enterococcus. In: Collier L, Balows A, Sussman M, eds. Microbiology and microbial infections. 9th ed. London: Edward Arnold 1997; 669-82.

13. Titze-De-Almeida R., Rollo Filho M., Silveira C.A.N., Rodrigues I.P., Fonsecaii R.F., Moraes L.M.P., Felipe M.S.S., Eudes Filho J., Nascimento R.S., Boelens H., Belkum A.V. Molecular epidemiology and antimicrobial susceptibility of Enterococci recovered from Brazilian intensive care units. Braz J Infect Dis 2004, 8 (3): 197-205.

14. Clinical and Laboratory Standard Institute - CLSI. Methods for dilution antimicrobial susceptibility test for bacteria that grow aerobically. Approved standard $15^{\text {th }}$ CLSI document M7-A7. Wayner, PA: CLSI/NCCLS, 2006.

15. Ostrowsky B. E.; Venkataraman L.; D’Agata, E. M. C. D. et al. Vancomycin-resistant enterococci in Intensive Care Units. Arch Intern Med 1999; 159:1467-72.
16. Bonten, M.K.M.; Willems, R.; Weinstein, R.A. Vancomycinresistant enterococci: why are they here, and where do they come from? Lancet Infect Dis 2001; 1:314-25.

17. Warren, D. K.; Kollef, M. H.; Seiler, S. M. et al. The epidemiology of vancomycin-resistant Enterococcus colonization in a medical Intensive Care Unit. Infect Control Hosp Epidemiol 2003; 24:257263.

18. Fridkin S.K.; Edwards J. R.; Courval, J. M. et al. The effect of vancomycin and third-generation cephalosporins on prevalence of vancomycin-resistant enterococci in 126 U.S. adult Intensive Care Units. Ann Intern Med 2001; 135:175-83.

19. Brown, D. F. J.; Walpole, E. Evaluation of selective and enrichment media for isolation of glycopeptide-resistant enterococci from faecal specimens. J Antimicrob Chemother 2003; 51:289-96.

20. Ledeboer N.A.; Tibbetts, R.J., Dunne, W.M. A new chromogenic agar medium, chromID VRE, to screen for vancomycin-resistant Enterococcus faecium and Enterococcus faecalis. Diagn Microbiol Infect Dis 2007; 59:477-9.

21. Dutka-Malen S., Evens S., Couvarlin P. Detection of glycopeptide resistance genotypes and identification to the species level of clinically relevant Enterococci by PCR. J Clin Microb 1995; 33(1): 24-7.

22. Woodford N., Egelton C.M., Morrison D. Comparison of PCR with phenotypic methods for the speciation of enterococci. Adv Exp Med Biol 1997; 418:405-8.

23. Kariyama R., Mitsuhata R., Chow J.W., et al. Simple and reliable multiplex PCR assay for surveillance isolates of vancomycin resistant enterococci. J Clin Microbiol 2000; 38:3092-5.

24. Zanella, R.C.; Brandileone, M.C.; Bokermann, S. et al. Phenotypic and genotypic characterization of vanA Enterococcus isolated during the first nosocomial outbreak in Brazil. Microb Drug Res 2003; 9:283-291.

25. Palladino S.; Kay I.D.; Flexman, J.P. Rapid detection of vanA and vanB genes directly from clinical specimens and enrichment broths by real-time multiplex PCR assay. J Clin Microbiol 2003; 41:2483-6. 